\title{
Ultrafast nanoelectronics: steering electrons in infrared near-fields (Conference Presentation) (Withdrawal Notice)
}

Georg Herink, Claus Ropers

Georg Herink, Claus Ropers, "Ultrafast nanoelectronics: steering electrons in infrared near-fields (Conference Presentation) (Withdrawal Notice)," Proc. SPIE 9547, Plasmonics: Metallic Nanostructures and Their Optical Properties XIII, 95470D (12 February 2018); doi: 10.1117/12.2190376

SPIE Event: SPIE Nanoscience + Engineering, 2015, San Diego, California, United States 


\section{Ultrafast nanoelectronics: steering electrons in infrared near-fields (Conference Presentation) (Withdrawal Notice)}

Proc. SPIE 9547, 95470D (2015)

Online Publication Date: 5 October 2015

Withdrawn from Publication: 12 February 2018

Conference Date: 9-13 August 2015

Conference Location: San Diego, California, United States

Conference Title: Plasmonics: Metallic Nanostructures and Their Optical Properties XIII

Conference Chairs: Allan D. Boardman, Din Ping Tsai

Georg Herink, Claus Ropers

Georg-August-Univ. Göttingen (Germany)

Publisher's Note: This conference presentation, originally published on 5 October 2015, was withdrawn per author request. 